\title{
JPR \\ UNDERGRADUATERESEARCH
}

\section{STEM}

\section{Development of a Scalable Synthesis of HP- $\beta$-CD Pluronic Polyrotaxanes}

\section{Student researchers: Joseph L. Skulsky, Senior, and Elizabeth A. Slepko, Junior}

Polyrotaxanes are polymers that have macrocycles threaded onto them, analogous to beads threaded onto a string. These materials are used for a variety of biomedical applications. The Thompson Group has been developing 2-hydroxypropyl- $\beta$-cyclodextrin (HP- $\beta-C D$ ) polyrotaxanes as therapeutics for the treatment of Niemann-Pick Type C (NPC) disease, a debilitating genetic disorder in which unesterified cholesterol accumulates in the lysosomes of cells. Developing a scalable process is crucial for the advancement of these materials as NPC therapeutics. The goal of this project is to optimize a protocol for the synthesis of HP$\beta-\mathrm{CD} /$ Pluronic polyrotaxanes in order to develop a method that can be operated on the multi-gram scale to support preclinical studies. Each component of the protocol was screened to determine which combination led to the formation of polyrotaxanes with the highest yields and threading efficiencies in the shortest amount of time. Threading efficiency is a measure of how many HP- $\beta$ $\mathrm{CD}$ molecules are threaded onto each polymer. Nuclear magnetic resonance (NMR) spectroscopy suggested that probe sonication and bath sonication are both necessary components of the optimal protocol. This indicates that sufficient agitation of the reaction mixture is required to promote the non-covalent threading reaction. Furthermore, bath sonication for one hour, followed by stirring for two days, gave the highest threading efficiency.

The results of these studies have streamlined the existing protocol for HP- $\beta-C D$ and Pluronic F127 and for HP- $\beta-C D$ and Pluronic L81; however, additional studies are needed to reveal whether this protocol is robust enough for efficient preparation of other HP- $\beta$ $\mathrm{CD} /$ Pluronic polyrotaxane derivatives.
Research advisor David H. Thompson writes: "The students worked tirelessly in the David H. Thompson Lab in the Purdue Department of Chemistry to prepare a family of 'wheel-and-axle' type of polymers known as polyrotaxanes. These compounds are of great interest because of their capacity to serve as cholesterolmobilizing molecules in Niemann-Pick Type C disease, as well as potentially acting as long circulating MRI agents for tumor detection. The students' work has laid an important foundation for us to pursue these applications by working out the details of their efficient synthesis."

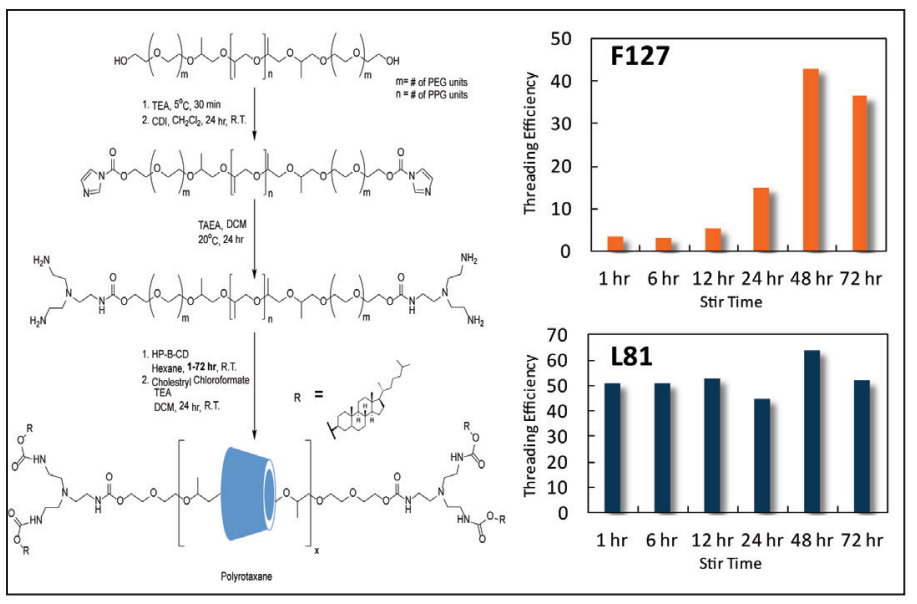

Synthesis of HP- $\beta-C D /$ Pluronic polyrotaxanes with cholesterol end-caps. The Pluronic triblock copolymer starting material is modified so that cholesterol end-caps can be used to keep the HP- $\beta$-CD molecules (blue structure) threaded. It was found that stirring the Pluronic F127 and HP- $\beta$-CD for 48 hours afforded the best threading efficiency, while highly threaded HP $\beta-C D /$ L81 polyrotaxanes were obtained after just 1 hour of stirring. 